\title{
Noise characteristics of stacked CMOS active pixel sensor for charged particles
}

\author{
Takuya Kunihiro $^{\mathrm{a}, *}$, Kazuhide Nagashima ${ }^{\mathrm{a}}$, Isao Takayanagi ${ }^{\mathrm{b}}$, \\ Junichi Nakamura ${ }^{\mathrm{b}}$, Koji Kosaka ${ }^{\mathrm{c}}$, Hisayoshi Yurimoto ${ }^{\mathrm{a}}$ \\ ${ }^{a}$ Department of Earth and Planetary Sciences, Tokyo Institute of Technology, 2-12-1 Ookayama, Meguro, Tokyo 152-8551, Japan \\ ${ }^{\mathrm{b}}$ Olympus Optical Co., Ltd., 2-3 Kuboyamacho, Hachioji, Tokyo 192-8512, Japan \\ ${ }^{\mathrm{c}}$ Tokyo Technology Co., Ltd., 446-8 Katakura, Hachioji, Tokyo 192-0914, Japan
}

Received 28 August 2000; received in revised form 30 November 2000; accepted 1 December 2000

\begin{abstract}
The noise characteristics of a stacked CMOS active pixel sensor (SCAPS) for incident charged particles have been analyzed under $4.5 \mathrm{keV} \mathrm{Si}^{+}$ion irradiation. The source of SCAPS dark current was found to change from thermal to electron leakage with decreasing device temperature. Leakage current at charge integration part in a pixel has been reduced to 0.1 electrons $\mathrm{s}^{-1}$ at $77 \mathrm{~K}$. The incident ion signals are computed by subtracting reset frame values from each frame using a non-destructive readout operation. With increase of irradiated ions, the dominant noise source changed from read noise, and shot noise from the incident ions, to signal frame fixed-pattern noise from variations in sensitivity between pixels. Pixel read noise is equivalent to ten incident ions. The charge of an incident ion is converted to 1.5 electrons in the pixel capacitor. Shot noise corresponds to the statistical fluctuation of incident ions. Signal frame fixed-pattern noise is $0.7 \%$ of the signal. By comparing full well conditions to noise floor, a dynamic range of $80 \mathrm{~dB}$ is achieved. SCPAS is useful as a two-dimensional detector for microanalyses such as stigmatic secondary ion mass spectrometry. (C) 2001 Elsevier Science B.V. All rights reserved.
\end{abstract}

PACS: $07.77 . \mathrm{Ka} ; 07.81 .+\mathrm{a} ; 87.66 . \mathrm{Pm} ; 82.80 . \mathrm{Ms}$

Keywords: Detector; Solid-state image sensor; Charged particle; Noise; SIMS

\section{Introduction}

An integral-type solid-state image sensor for charged particles, termed a stacked active pixel sensor (APS), has recently been developed [1-4]. The operational principle of APS is based on detecting the change in potential of a floating

\footnotetext{
*Corresponding author. Tel.: +81-35734-2243; fax: +8135734-3538.

E-mail address: kunihiro@geo.titech.ac.jp (T. Kunihiro).
}

capacitor, as caused by charged particle irradiation. The APS is composed by pixel array and scanning electronics. As the pixel array consists of $512 \times 490$ rectangle micro-detectors, simultaneous two-dimensional detection for charged particles and/or high energy particles is achieved. The effectiveness of the APS has been demonstrated for ions [1,5], electrons [6,7] and soft X-rays [8].

APS has several advantages over conventional systems, such as two-dimensional detection, a wide dynamic range, no period of insensitivity, the 
direct detection of charged particles, constant ion sensitivity for all nuclides, and high robustness. APS is applicable to two-dimensional microanalysis, particularly to isotope analysis using stigmatic secondary ion mass spectrometry. The isotope ratios of certain elements in such analyses range over six orders of magnitude, and yet the relative isotopic variation in nature is less than several per cent. Thus, the two-dimensional detection of isotope ratios over six orders of magnitude with consistent accuracy is necessary for the precise determination of isotopic ratios in natural samples. Integrating detectors capable of storing charge are suitable for precise low-intensity measurements given a sufficient time period for integration, and can reduce statistical errors. As APS has no period of insensitivity and is very robust against ions, it is possible to measure incident charged particles with both high and low intensities, distributed in two dimensions, in the same time interval and under the same analytical conditions without danger of mechanical or electronic damage, although conventional pulse count detectors are limited with regard to beam intensity.

The output and noise characteristics of detectors are important in determining the dynamic range of incident charged particles detection and detection limits. The output characteristics of a stacked CMOS-type APS (SCAPS) for charged particles was evaluated, and a linearity correction method has been established [4]. The aim of this study is to demonstrate the noise characteristics of SCAPS for charged particles.

\section{Basic description of SCAPS}

A SCAPS pixel unit consists of a pixel capacitor $C_{\text {PIX }}$ and three transistors; a readout MOS transistor $M_{\mathrm{RD}}$, a reset MOS transistor $M_{\mathrm{RS}}$ and a row selection switch $M_{\mathrm{Y}}$ (Fig. 1). During operation, the pixel electrode which exists at a top surface is charged by incident charged particles. The charge on the electrode is proportional to the number of charged particles. The carriers generated are integrated via the pixel capacitor $C_{\text {PIX }}$. The potential of the pixel electrode $V_{\mathrm{PIX}}$ is reset to voltage $V_{\mathrm{RS}}$ using the $M_{\mathrm{RS}}$. The

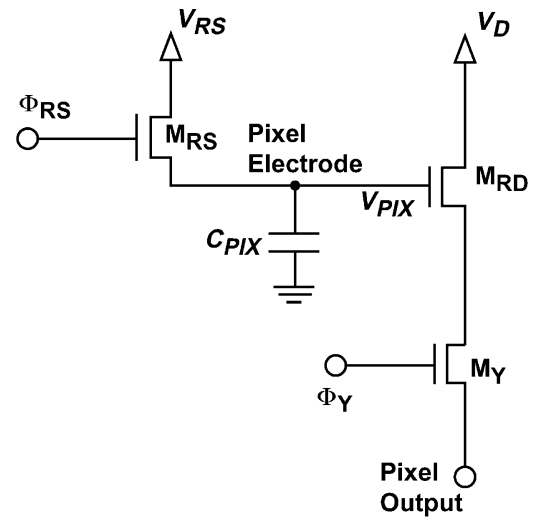

Fig. 1. Schematic circuit layout of a pixel unit of SCAPS.

potential $V_{\text {PIX }}$ then changes as a result of charged particle-induced secondary-electron emission and particles incident on the pixel electrode directly. The pixel signal is output through the row selection switch $M_{\mathrm{Y}}$. The change in potential $V_{\mathrm{PIX}}$ is read out by a voltage follower circuit which consists of $M_{\mathrm{RD}}$ and an external load resistor which is described below. As the input impedance of the readout transistor $M_{\mathrm{RD}}$ is very high, the signal charges stored in $C_{\text {PIX }}$ can be readout multiple times non-destructively until the following reset operation. Signal readout with and without the reset operation is called destructive readout (DRO) and non-destructive readout (NDRO), respectively.

The SCAPS is designed with pixel dimensions of $14 \mu \mathrm{m}(\mathrm{H}) \times 14 \mu \mathrm{m}(\mathrm{V})$, and pixel electrode dimensions of $12 \mu \mathrm{m}(\mathrm{H}) \times 12 \mu \mathrm{m}(\mathrm{V})$, yielding a fill factor of $73 \%$. The imaging area is an array of $512 \times 490$ independent pixels, and measures $7.17 \mathrm{~mm}(\mathrm{H}) \times 6.86 \mathrm{~mm}(\mathrm{~V})$. The pixel signal is output as potential $V_{\text {OUT }}$ through an off-chip load resistor $\left(R_{\mathrm{L}}=20 \mathrm{k} \Omega\right)$ at the end of the horizontal signal line forming a source follower of the readout transistor $M_{\mathrm{RD}}$ inside the pixel. A detailed description is provided by Nagashima et al. [4].

\section{Experimental}

A schematic diagram of an ion detection system is illustrated in Fig. 2. The system is a secondary ion mass spectrometry (SIMS) with a SCAPS 


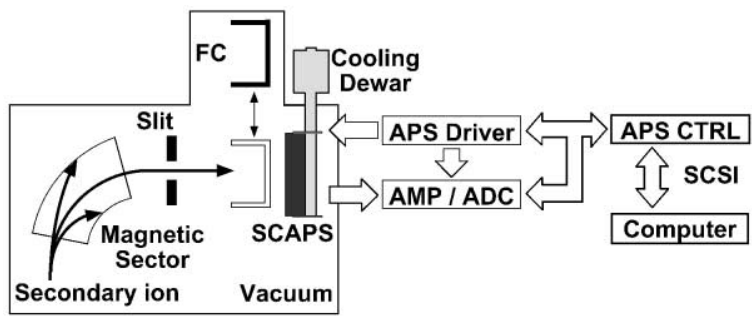

Fig. 2. Schematic diagram of experimental system for ion irradiation.

attachment. The charged particles that irradiated the SCAPS were $4.5 \mathrm{keV}$ accelerated ions. The ion beam was generated by a CAMECA IMS- $3 f$ SIMS instrument. The SCAPS was positioned on the secondary ion image plane. The image plane was held at a vacuum of $0.5 \mu \mathrm{Pa}$. The ion signal from the SCAPS after ion irradiation was output at a scan rate of $20 \mathrm{kHz}$ per pixel by adding driving pulses to the SCAPS from the APS driver. The storage time for the ion signals can be chosen to be $20 \mathrm{~s}$ and above. The signals from each pixel are amplified by a low-noise amplifier (Analog Devices, SSM-2017) and converted into a digital signal by a 16-bit A/D converter (Datel, ADS930MC) in an external readout circuit, and the digital signals were processed by computer. In order to reduce environmental noise, the SCAPS device was linked to the electronics by differential signal lines using shielded twisted pair cables through an ultra-high vacuum seal.

The raw output signal from a pixel is not precisely proportional to the number of electrons in $C_{\text {PIX }}$. Therefore, linearity correction as proposed by Nagashima et al. [4] was applied to the raw output. The corrected value has arbitrary units and is proportional to the number of electrons in $C_{\text {PIX }}$.

In order to control dark current in a pixel and offset drift of a pixel output during ion irradiation and during non-irradiated processing time, the temperature of the device was maintained at a constant cool temperature using Dewar. The temperature was monitored using a chromelalumel thermocouple attached to the front of the SCAPS package.

In order to determine noise characteristics as a function of incoming ions, a uniform secondary ion beam was used to irradiate the SCAPS. A uniform secondary ion beam distribution was achieved by irradiating a $250 \mu \mathrm{m} \times 250 \mu \mathrm{m}$ area on a silicon wafer with a small rastered primary beam of ${ }^{16} \mathrm{O}^{-}$ions; the secondary ${ }^{28} \mathrm{Si}^{+}$or ${ }^{30} \mathrm{Si}^{+}$ ions generated in a $60 \mu \mathrm{m}$ diameter central area of the rastered primary-beam area were projected directly onto the SCAPS imaging area using the stigmatic ion optics of the SIMS. The projected area covered about $40 \%$ of the central field of the SCAPS imaging area, which corresponds to about $10^{5}$ pixels. The total count rate of secondary ions projected onto the imaging area was measured by inserting the faraday cup (FC) of the IMS- $3 f$. The incident ion rate for a single pixel was calculated by dividing the FC intensity by the number of irradiated pixels.

\section{Definition of SCAPS noise}

The raw signal image output from a solid state imager includes two kinds of noises; random noise and fixed-pattern noises [9]. The random noise in SCAPS imaging is composed of read noise, shot noise and reset noise. Read noise originates from the internal and external readout circuits of the device, shot noise is caused by dark current and statistical fluctuation of incoming ions, and reset noise occurs during the reset operation.

Dark current is a signal generated within a device in the absence of operational input [10]. It is supposed that dark current in SCAPS is caused by thermal generation current and by gate leakage current in the pixel. Thermally generated electronhole pairs in the semiconductor junction between source and substrate of $M_{\mathrm{RS}}$ contribute charge to the capacitor. The temperature dependence of thermal dark current is expressed by

$J_{T}=C \exp \left[\frac{-E_{\mathrm{G}}}{2 k T}\right]$,

where $E_{\mathrm{G}}, C, k$ and $T$ are the activation energy typically corresponding to the band gap of the device material, a constant, Boltzmann's constant, and temperature of the device, respectively [10]. Thermal generation current strongly depends on temperature. Gate leakage current of $M_{\mathrm{RS}}$ causes 
dark current, too. Especially in low operating temperature, gate leakage assisted by host carriers becomes sufficient because thermal generation current is extremely reduced at low temperatures [11].

The number of electrons generated due to dark current is random and obeys a Poisson distribution. Thus, shot noise occurs. If dark current is not sufficiently reduced, then the shot noise over long exposure times will increase the overall background noise and ultimately limit the system sensitivity.

In the device operation, pixel electrode should be initialized to reset bias $V_{\mathrm{RS}}$ before charge integration through reset transistor $M_{\mathrm{RS}}$ (Fig. 1). Uncertainties introduced by this scheme will be caused by the thermal noise of the resistance through which the floating gate is reset. This is called reset noise and is independent of time and resistance [12]. Reset noise introduces a random distribution of reset voltages among pixels and affects the quality of imagery. The theoretical standard deviation of reset noise $\sigma_{\mathrm{rs}}$ for a pixel is expressed in units of electron number as

$\sigma_{\mathrm{rs}}=\frac{\sqrt{k T C_{\mathrm{PIX}}}}{e}$,

where $C_{\text {PIX }}$ is the capacitance of a pixel. From this equation, using a pixel capacitance of $15.8 \mathrm{fF}$, as estimated from the layout and process parameters of SCAPS, the reset noise of the SCAPS at $77 \mathrm{~K}$ is estimated to be about 26 electrons [3].

SCAPS consists of 250,000 independent pixels, and the output characteristics of each pixel are not strictly the same [4]. Thus, variation in the output characteristics of pixels acts like noise in reproduced images. As this kind of noise is fixed with the location of the pixel and is independent of time, the noise caused by variations in the output characteristics of pixels is called fixed-pattern noise (FPN). Theoretically, the output characteristics of a pixel are expressed as

$N_{\mathrm{e}}^{i}=\frac{C_{\mathrm{PIX}}^{i}}{e}\left(V_{\mathrm{OUT}}^{i}+V_{\mathrm{TH}}^{i}+\sqrt{\frac{V_{\mathrm{OUT}}^{i}}{a^{i} R_{\mathrm{L}}}}\right)$,

where $i$ is a pixel numerical identifier, $N_{\mathrm{e}}$ is the number of electrons in a pixel capacitor, $V_{\mathrm{TH}}$ is the threshold voltage of the read MOS transistor of the SCAPS, and $a$ is a coefficient dependent on pixel size, electron mobility, and capacitance of gate oxide [4]. Non-uniformity of $V_{\mathrm{TH}}$ of pixels causes noise which corresponds to differences of output signals in the absence of ion irradiation (reset frame FPN). On the other hand, nonuniformity of $a$ of pixels causes noise which corresponds to sensitivity variation with ion irradiation (signal frame FPN) .

\section{Results and discussion}

\subsection{Estimation of number of electrons in $C_{\mathrm{PIX}}$}

The change in $\eta$ after reset operation $\Delta \eta$ was measured over time at $77 \mathrm{~K}$ without ion irradiation under NDRO operation (Fig. 3). The $\Delta \eta$ plotted in Fig. 3 is an average value calculated from a $11 \times 11$ pixel SCAPS sub-array. From the figure it can be seen that change of $\Delta \eta$ is large in several frames immediately following the reset operation, and then becomes smaller and constant after that. This peak is due to the non-steady readout state of SCAPS. Steady state readout is achieved after several frames following the reset operation. Under the steady state readout conditions, $\Delta \eta$ increases

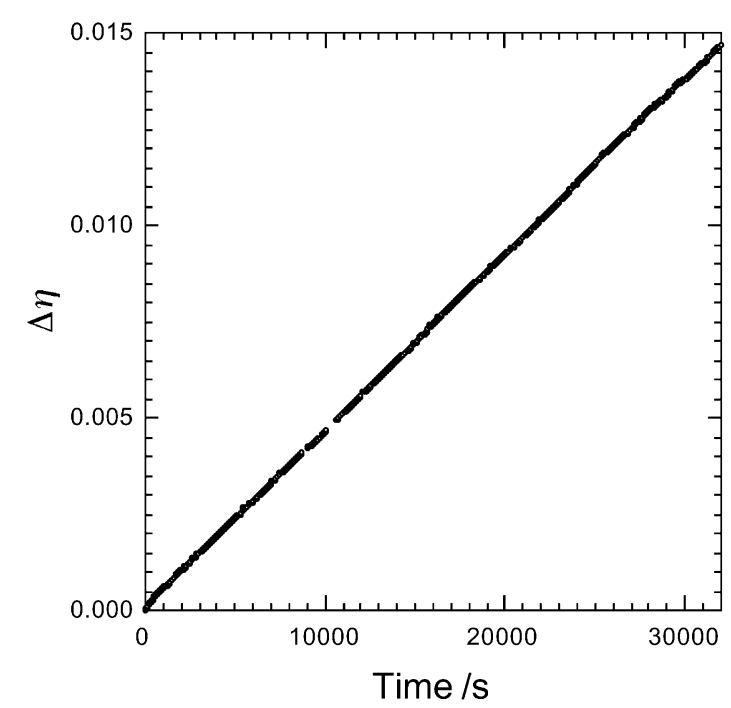

Fig. 3. Variation of dark signal with time. $\Delta \eta$ : output of a pixel after linearity correction. 


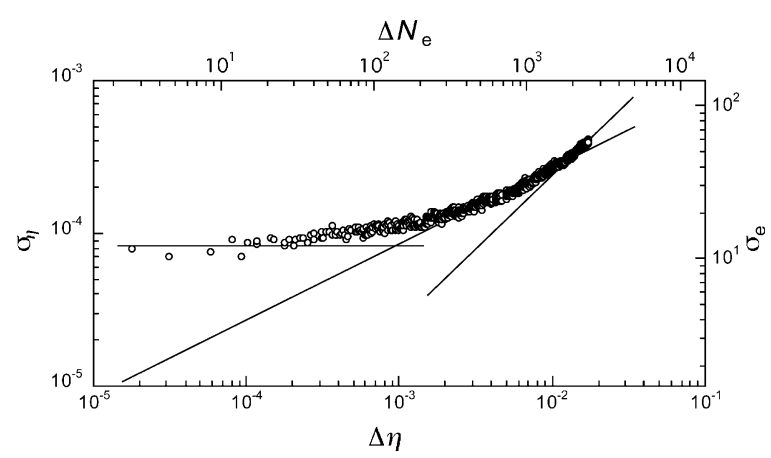

Fig. 4. Relationship between dark signal variation and the standard deviation. $\Delta \eta$ : average of signal of a pixel after linearity correction, $\sigma_{\eta}$ : standard deviation of $\Delta \eta, \Delta N_{\mathrm{e}}$ : number of electrons in a pixel, $\sigma_{\mathrm{e}}$ : standard deviation of $\Delta N_{\mathrm{e}}$.

linearly with time due to dark current. The relationship between $\Delta \eta$ and the standard deviation among the sub-array $\sigma_{\eta}$ is shown in Fig. 4. As $\Delta \eta$ increases, the slope of the line changes from 0 , to $\frac{1}{2}$ and then 1 . The dominant noise under conditions of slopes $=0$ or 1 are circuit read noise and dark current FPN, respectively. The dominant noise in the slope $=\frac{1}{2}$ region is dark current shot noise.

As dark current obeys the Poisson law, the shot noise of dark current can be expressed as

$\sigma_{\mathrm{e}}^{2}=\Delta N_{\mathrm{e}} \propto \Delta \eta$,

where $\sigma_{\mathrm{e}}$ is the standard deviation of shot noise and $\Delta N_{\mathrm{e}}$ is the electron number generated by dark current. According to this relationship, the arbitrary unit of $\eta$ is converted into an electron number. The conversion scale is indicated on the upper and right axis of Fig. 4. The SCAPS signal is expressed as an equivalent number of electrons in $C_{\text {PIX }}$ in this paper.

\subsection{Temperature dependence of dark current}

The average dark current of the $11 \times 11$ subarray of pixels of SCAPS over a temperature range of 282 to $77 \mathrm{~K}$ has been measured (Fig. 5).

There are two distinct temperature dependencies of dark current. In the higher temperature region, dark current decreases according to

$$
\frac{\mathrm{d} N_{\mathrm{e}}}{\mathrm{d} t}=3.5 \times 10^{12} \exp \left[-\frac{1.1(\mathrm{eV})}{2 k T}\right]\left(\mathrm{s}^{-1}\right) \text {. }
$$

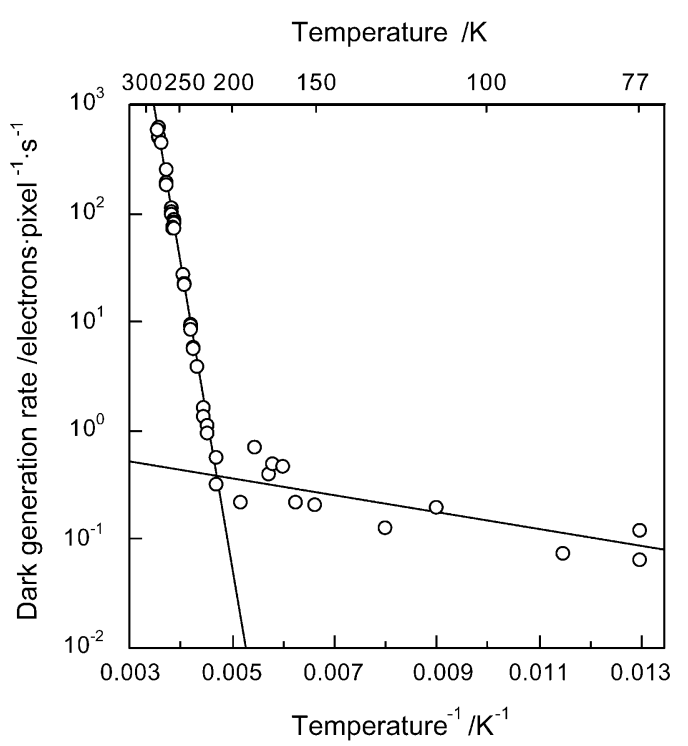

Fig. 5. Dark current of SCAPS as a function of temperature.

The activation energy is in good agreement with the band gap of silicon. According to Eq. (1), the higher temperature region is thought to be dominated by thermal dark current. In the lower temperature region, dark current decreases according to

$\frac{\mathrm{d} N_{\mathrm{e}}}{\mathrm{d} t}=0.9 \exp \left[-\frac{0.031(\mathrm{eV})}{2 k T}\right]\left(\mathrm{s}^{-1}\right)$.

The activation energy at lower temperatures is smaller than that at higher temperatures. The lower temperature region is thought to be dominated by electron leakage dark current. The number of electrons per pixel due to the dark current at $77 \mathrm{~K}$ is estimated to be approximately $0.1 \mathrm{~s}^{-1}$.

The decrease in signal and shot noise caused by dark current may cause problems in integration measurements over long periods. In this study, the temperature of the SCAPS was held constant at $77 \mathrm{~K}$ using liquid nitrogen in the Dewar (except for experiments of temperature dependence). The longest integration period was $350 \mathrm{~s}$. Thus, the maximum decrease in signal and shot noise by dark current are 35 and 6 electrons, respectively.

\subsection{Reset frame FPN}

The heterogeneity of the SCAPS reset frame is shown in Fig. 6a. The standard deviation of the 
reset frame FPN is 600 electrons, which correspond to $0.43 \%$ referenced to the saturation signal level. The degree of reset frame FPN for SCAPS in reference to the saturation signal level is less than that $(1.1 \%)$ of AMI [1]. This demonstrates that the new CMOS design stabilizes the device and reduces reset frame FPN to a third of that of AMI.

As the principle of SCAPS is the sensing of the potential shift of a floating photodiode caused by ion-induced secondary-electron emission and incident ion, signal information corresponding to incident particles is the change of output between signal and reset frames. Eq. (3) indicates that subtraction of the reset frame from the signal frame can eliminate the reset frame FPN. We define this operation as a reset frame correction. Non-steady state readout occurs for several frames after the reset operation, and hence a frame under steady state readout conditions was selected as a reset frame.

The effect of reset frame correction for the SCAPS device is shown in Fig. 6. Each image is a reproduction of a $4.5 \mathrm{keV}^{27} \mathrm{Al}^{+}$image of a process pattern mounted on a silicon wafer. The signal integration time was $20 \mathrm{~s}$ and the average rate of induced ${ }^{27} \mathrm{Al}^{+}$secondary ions per pixel was 20 counts/pixel. In Fig. 6a, ion images are difficult to recognize because the reset frame FPN is larger than the ion signal. After reset frame correction, the reset frame FPN is suppressed and a secondary ion image becomes apparent (Fig. 6b). The horizontal intensity changes between the arrows of each image are shown in a diagram below each image.

\subsection{Ion transfer and noise performance with ion irradiation}

The ion transfer and total noise characteristics at $77 \mathrm{~K}$ were evaluated by determining the average and standard deviation of signals, respectively, from a typical $11 \times 11$ sub-array. The incident ions were uniformly irradiated on the sub-array. The ion transfer characteristics and total noise characteristics after reset frame correction under DRO and under NDRO were measured as a function of
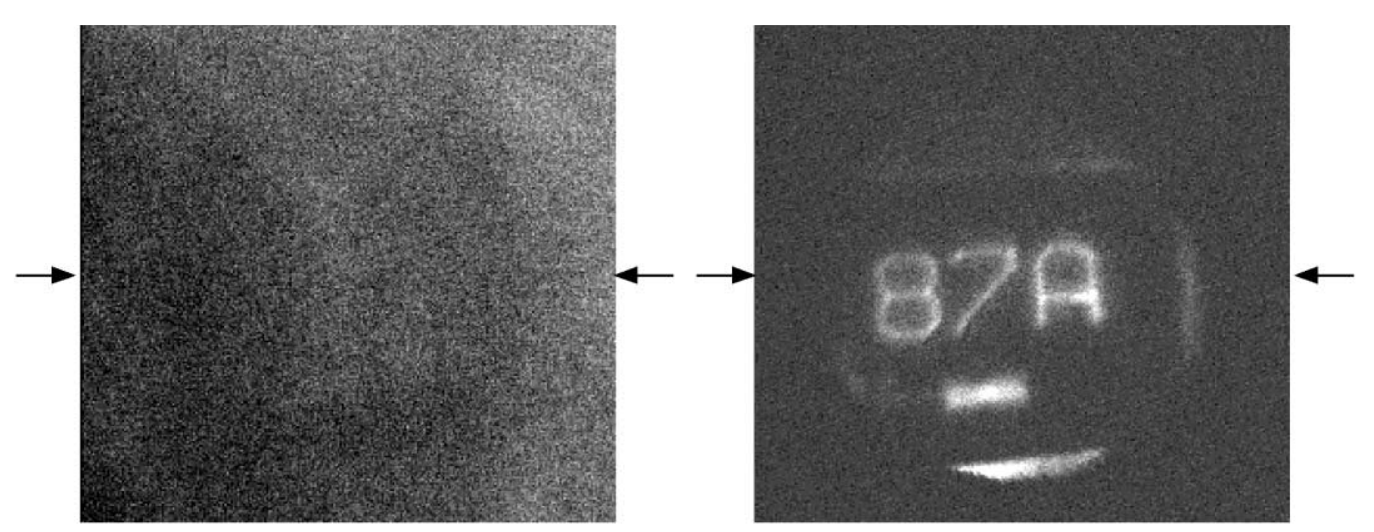

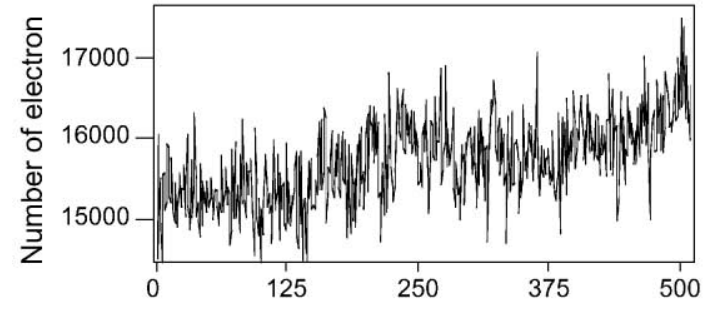

(a)

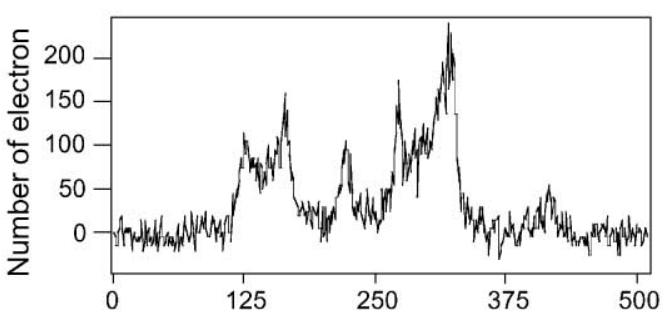

(b)

Fig. 6. (a) Raw image after irradiation of a $4.5 \mathrm{keV} \mathrm{Al}^{+}$ion image of a process pattern on a silicon wafer. (b) Calibration image after reset frame correction. 


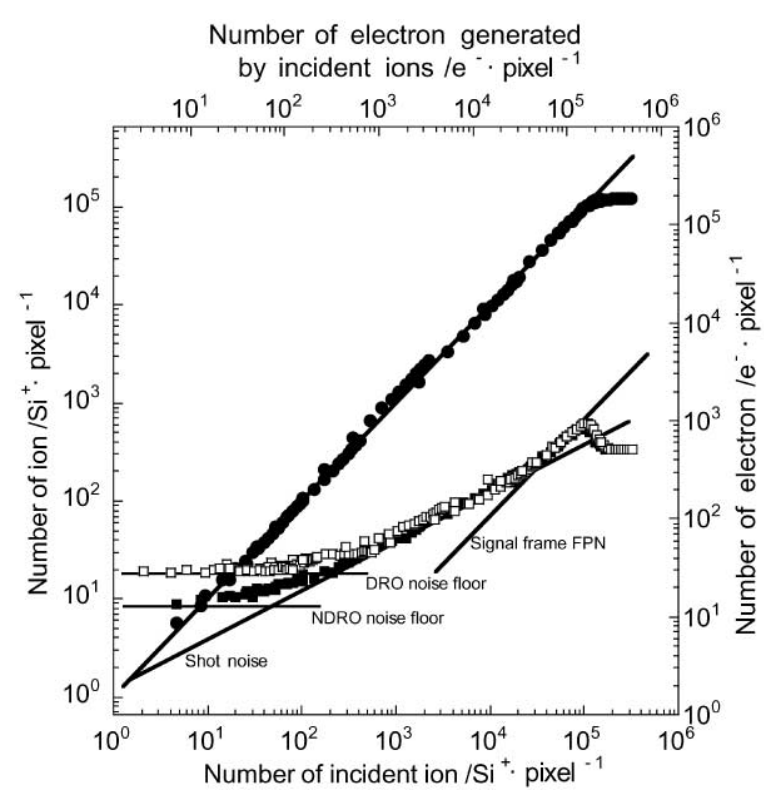

Fig. 7. Ion transfer and the noise characteristics against incident $\mathrm{Si}^{+}$ions.

the number of incident ions (Fig. 7). Solid circles represent the ion transfer performance of the SCAPS, and open and solid rectangles represent noise performance by DRO and NDRO, respectively.

The ion transfer plot shows that the operating range of SCAPS spans more than four orders of magnitude. The electron accumulation rate in $C_{\text {PIX }}$ is 1.5 for a single ion irradiation of $4.5 \mathrm{keV} \mathrm{Si}^{+}$. The saturation signal level of a pixel was observed to be $1.5 \times 10^{5}$ electrons. As the number of electrons in $C_{\text {PIX }}$ in the reset frame is estimated to be $2 \times 10^{4}$ electrons, the full well condition of $C_{\text {PIX }}$ of the SCAPS is $1.7 \times 10^{5}$ electrons.

There are three principal domains of noise in imaging; the noise floor regime, shot noise regime, and pixel non-uniformity regime with high incident ion increase (Fig. 7). As the longest integration period in this experiment was $350 \mathrm{~s}$ and the device temperature was maintained at $77 \mathrm{~K}$, the shot noise caused by dark current was less than 6 electrons. Dark current correction was not applied to the data, because the level of noise can be considered to be negligibly small for the discussion below.
The noise floor in DRO operation was 29 electrons. The DRO noise floor is associated with read noise and reset noise, as mentioned in the definition of SCAPS noise section. The noise level corresponds to about 20 incident ions. After cancelling the reset noise by NDRO operation, the noise floor of NDRO became 13 electrons, which is equivalent to about 10 incident ions. By comparing the noise floors between DRO and NDRO, the reset noise component is calculated to be 23 electrons, which is in excellent agreement with the theoretical value of 26 electrons.

The noise floor in NDRO consists primarily of read noise from the internal and external readout circuits of the device. The read noise of the external readout circuit, which consists of an offchip load resistor, amplifier and a 16-bit A/D converter, was measured under the same operating conditions of SCAPS readout with ground level monitoring. The measured read noise of the external readout circuit was equivalent to $3 \mathrm{elec}-$ trons, and was dominated by quantization resolution in the $A / D$ converter. Therefore, the noise of the internal device readout circuit, which consists of a readout MOSFET, is estimated to be 13 electrons. The dynamic range of a pixel, as defined by the ratio of the saturation level to the noise floor in NDRO, is $80 \mathrm{~dB}$.

As the incident signal increases, noise becomes dominated by shot noise from the incident ions. This is the intermediate region of the noise curve in Fig. 7 and is characterized by a line of slope $\frac{1}{2}$. The slope is a result of the statistical fluctuation of incident ions, and the uncertainty in the output of SCAPS is proportional to the square root of the number of incident ions because the intensity of incident ions obeys the Poisson law. This noise is inherent and cannot be removed. The extrapolated curve of the shot noise of incident ions crosses the ion transfer curve at a point at which the number of incident ions per pixel is 1.5. Theoretically, the crossing point should be at 1 ion per pixel. This discrepancy is due to neglecting $73 \%$ pixel fill factors in our calculations of the number of incident ions. If these fill factors are considered in the calculation, the crossing point moves to the theoretical value within experimental error. 
In the pixel non-uniformity regime, noise is linearly dependent on the signal. This noise is caused, in particular, by variations in the pixel sensitivity between pixels, and is termed signal frame FPN. The sensitivity characteristics of pixels should be associated to variations of semiconductor fabrication processes. Therefore, each pixel has a unique collecting dimension, MOSET readout gain and quantum efficiency. Signal frame FPN is calculated to be $0.7 \%$ referenced to the signal level, which is almost equivalent to $0.5 \%$ by AMI [1].

\section{Conclusions}

The noise properties of SCAPS have been evaluated using $4.5 \mathrm{keV}{ }^{28} \mathrm{Si}^{+}$and ${ }^{30} \mathrm{Si}^{+}$ion irradiation. Thermal dark current is dominant at temperatures exceeding $230 \mathrm{~K}$. Below $230 \mathrm{~K}$, the dominant dark current changes to electron leakage. The dark current at $77 \mathrm{~K}$ is 0.1 electrons $\mathrm{s}^{-1}$. The reset frame FPN is $0.4 \%$ referenced to the pixel saturation level. Reset frame FPN and reset noise are cancelled completely under NDRO operation. As the number of incident ions increases, the dominant noise source changes from read noise and shot noise of incident ions, to signal frame FPN. The charge of a single incident ion is converted to 1.5 electrons in $C_{\text {PIX }}$. The read noise at $77 \mathrm{~K}$ is 13 electrons, which corresponds to 10 incident ions. The full well condition of $C_{\mathrm{PIX}}$ is estimated to be $1.7 \times 10^{5}$ electrons. The dynamic range achieved by SCAPS, as defined by the difference between the read noise and the full well condition of $C_{\mathrm{PIX}}$, is over $80 \mathrm{~dB}$. The level of shot noise is consistent with the statistical fluctuation of incident ions in a pixel after fill factor correction. The noise characteristics of SCAPS make the device suitable for use as a sensor in the twodimensional simultaneous analysis of charged particles.

\section{Acknowledgements}

We are grateful to $H$. Emori for helpful comments. This study was partly supported by Monbu-Kagaku-sho and Gakushin-fellowship.

\section{References}

[1] K. Matsumoto, et al., IEEE Trans. Electron Devices 40 (1993) 82.

[2] I. Takayanagi, et al., Proc. 999 IEEE workshop on Charge-Coupled Devices and Advanced Image Sensors, 1999, p. 159

[3] I. Takayanagi, et al., Tech. Rep. IEICE, Vol. 99, 1999, p. 7 (in Japanese).

[4] K. Nagashima, et al., Surf. Interface Anal. 31 (2000) 131.

[5] H. Yurimoto, et al., in: A. Benninghoven, Y. Nihei, R. Shimizu, H.W. Werner (Eds.), Secondary Ion Mass Spectrometry SIMS IX, Wiley, Chichester, 1994, pp. 258-261.

[6] H. Yurimoto, et al., Bunseki Kagaku 45 (1996) 493 (in Japanese).

[7] H. Yurimoto, et al., in: A. Benninghoven, B. Hagenhoff, H.W. Werner (Eds.), Secondary Ion Mass Spectrometry SIMS X, Wiley, Chichester, 1997, pp. 987-990.

[8] I. Takayanagi, et al., IEEE Trans. Electron Devices 42 (1995) 1425.

[9] M. Blouke, et al., Opt. Eng. 22 (1983) 607.

[10] D.F. Barbe, Proc. IEEE 63 (1975) 38.

[11] E. Takeda, et al., Dig. Tech. Papers, Symp. on VLSI Technology, 1983, p. 104

[12] J.E. Carnes, et al., RCA Rev. 33 (1972) 327. 